\title{
A review of post infectious pulmonary fibrosis in the era of COVID-19 and potential treatment options
}

\author{
Medha Ghose MD, Maehali Patel MD
}

\begin{abstract}
On March 11, 2020, the World Health Organization (WHO) released a statement characterizing COVID-19 as a pandemic that has, as of October 2020, caused almost 36 million confirmed global cases and over 1 million deaths. One of the long-term complications suggested by researchers is fibrosis. It has been hypothesized that the combination of ongoing pulmonary injury caused by COVID-19 and the inability to promptly repair damage results in interstitial matrix widening and eventual compression and destruction of alveoli and capillaries. Here we focus on pathogenesis, risk factors, different infectious causes of fibrosis along with COVID-19, and potential treatment options that might reduce its effects.
\end{abstract}

Key words: COVID-19, pulmonary fibrosis, mechanism, treatment

\section{INTRODUCTION}

On March 11, 2020, the World Health Organization (WHO) released a statement characterizing COVID19 as a pandemic, ${ }^{1}$ a disease outbreak that has spread over multiple countries and continents. ${ }^{2}$ As of October 2020, there have been almost 36 million confirmed global cases and over 1 million deaths caused by this disease. ${ }^{3}$ COVID-19, or Coronavirus Disease 2019, is caused by SARS-CoV-2, a virus in the Coronaviridae family of viruses. These viruses usually cause upper respiratory tract illnesses, like the common cold. Most viruses in this family do not infect humans, and most of the seven coronaviruses known to infect humans cause only mild to moderate disease. However, three viruses can cause a more serious and potentially fatal disease. ${ }^{4}$ SARS coronavirus emerged in 2002 and was found to cause severe acute respiratory syndrome (SARS); MERS (Middle East respiratory syndrome) coronavirus emerged in 2012 and continues

Corresponding author: Medha Ghose

Contact Information: Medhaghose1692@gmail.com DOI: $10.12746 /$ swrccc.v9i40.883 to cause localized outbreaks around the world. The third deadly coronavirus is SARS-CoV-2. Because this disease is caused by a virus that belongs to a family of previously studied viruses, we can infer or hypothesize potential outcomes of the infection before we have the concrete studies needed to support theories. This paper will discuss the potential for lung fibrosis as a long-term complication in patients who have been infected by SARS-CoV-2.

\section{MECHANISM OF PULMONARY FIBROSIS}

Idiopathic pulmonary fibrosis (IPF) is the most common interstitial lung disease (ILD), a group of pulmonary disorders characterized by inflammatory changes in the alveoli that results in irreversible fibrosis and, subsequently, impaired lung function. It is likely caused by excessive replication of type II pneumocytes in response to alveolar damage or microinjuries, caused by several potential factors. In normal lungs, damage to type I pneumocytes that line the alveoli causes a sequence of changes starting with proliferation and differentiation of type II alveolar cells and stem cells and type II epithelial cells that restore the alveolar epithelium through activation of coagulation, angiogenesis, fibroblast activation and migration, 
collagen synthesis and proper alignment. These processes are performed through the different actions of the chemokines, such as TGF- $\beta$, platelet-derived growth factor (PDGF), fibroblast growth factor (FGF), and vascular endothelial growth factor (VEGF). In normal lungs, type II pneumocytes stimulate fibroblasts in the interstitium to become myofibroblasts in response to TGF-beta. These myofibroblasts produce reticular and elastic fibers, which are then secreted outside of the cell into the interstitium. The reticular fibers are responsible for structural support, while the elastic fibers give the lung its elasticity. The myofibroblasts then undergo apoptosis, and the cycle stops. .,6,8 $^{5,8}$

In fibrotic lungs, the alveolar repair cycle becomes irreversible. Micro injuries responsible for repetitive injuries to the type I pneumocytes render the type II pneumocytes dysfunctional, leading to functionally impaired lungs. ${ }^{7}$ Repetitive injuries to the lung trigger protein overexpression and imbalance between the cellular demand of protein synthesis and protein folding and maturation in the endoplasmic reticulum (ER), leading to misfolding of the proteins known as the "ER stress." Consequently, another pathway becomes activated, leading to not only inhibition of protein translation and targeting them for degradation but also causing apoptosis of the cells when the stress persists. This response is known as the unfolded protein response (UPR). This response, along with resulting in activation of intracellular apoptotic pathways, also induces the production of profibrotic mediators, such as TGF- $\beta 1$, PDGF, CXCL12 (C-X-C motif chemokine 12), and CCL2 (chemokine C-C motif ligand 2). ${ }^{5}$ Among those, TGF- $\beta 1$ is the most important factor that leads to alveolar apoptosis, epithelial-mesenchymal transition (EMT), epithelial cell migration, increased replication and activation of myofibroblasts in the interstitium, and production of VEGF and CTGF connectivetissue growth factor), including other profibrotic and pro-angiogenic mediators responsible for several other pathways. $^{8}$ The proinflammatory chemokines and cytokines produced by the damaged type I pneumocytes lead to several reactions in the lungs, including increased vascular permeability, new vessel formation, and endothelial cell proliferation, including endothelial progenitor cells (EPCs), which is a normal response to any injury. Malli et al. demonstrated that IPF patients have significantly reduced numbers of EPCs, resulting in failure of reendothelialization, formation of dysfunctional alveolar-capillary barriers, stimulating profibrotic response, and resultant augmentation of VEGF. This growth factor leads to abnormalities in vessel function, such as increased vascularization in the area surrounding the fibrotic foci, subsequently facilitating fibrogenesis. ${ }^{9,10}$ Consequently, the overproduction of collagen (e.g., reticular and elastic fibers) and thickening of the interstitium between the alveoli and the capillaries leads to ventilation-perfusion mismatch within the lungs and decreases lung compliance due to the excess collagen fibers. ${ }^{11}$ The restricted lung expansion causes changes in pulmonary function tests consistent with restrictive lung disease with decreased total lung capacity (TLC), forced vital capacity (FVC), and forced expiratory volume in 1 second (FEV1). ${ }^{12}$ The process of fibrosis is thought to be irreversible and progressive. The combination of ongoing pulmonary injury and the inability to promptly repair damage results in an interstitial matrix widening and eventual compression and destruction of alveoli and capillaries. This process substantially contributes to the respiratory failure that can occur within 3-7 years. $^{13}$

\section{RISK FACTORS}

Several risk factors have been implicated in the pathogenesis of IPF, including physiological aging leading to resistance to apoptosis along with increased fibrotic responses after injury with environmental stimuli, such as cigarette smoking and metal dust, agriculture, and farming, livestock, wood dust, stone, silica, sand, and microbial agents, such as pathogenic bacterial and viral infections. ${ }^{5}$ However, in recent years, genetic susceptibility and changes in gene expression of individuals developing IPF to environmental triggers have also become apparent. Rare genetic mutations have been found in the genes of IPF in adults, including surfactant protein C (SFTPC) and A2 (SFTPA2), leading to protein misfolding in the ER of type II pneumocytes and enhanced ER stress respectively. ${ }^{14}$

Researchers have also found rare variants in genes regulating telomerase and telomeraseassociated proteins, such as TERT and TERC that led 
to telomere shortening. ${ }^{15}$ In studies on changes in gene expression, transcriptional and translational changes in the profile of mRNA expression have also been found in the lungs of the IPF patients. Nance et al. in 2014 identified 873 differentially expressed genes in lung biopsies of eight IPF patients compared with seven controls, and 675 showed alternative splicing events in genes coding for periostin (POSTN) and collagen (COL6A3). ${ }^{16}$ Depianto et al. and Bridges et al. used microarrays to identify differential expression of 2940 and 781 genes, including genes encoding for growth factors, collagens, proteinases, and cytokines. ${ }^{17,18}$ Bridges et al. found the Twist1 gene, a gene with a protective function against apoptosis, was the most upregulated in IPF lungs. Studies were also done to assess gene expression that leads to acute exacerbations of IPF. Konishi et al. identified 579 differentially expressed genes associated with acute exacerbations with cyclin A2 (CCNA2) and $\alpha$-defensins being the most upregulated. ${ }^{19}$ Lung biopsies of patients with progressive IPF also showed differential expression of 243 transcripts, including C-C Motif Chemokine Ligand 2 (CCL2) and SFTPA1 when compared with lung biopsies from stable IPF patients. ${ }^{20}$

In addition to changes in gene expression, epigenetic alterations, such as DNA methylation, microRNA dysregulation, and histone modifications, have also been implicated in changes in the IPF lung. ${ }^{21}$ Yang et al. established a significant association between DNA methylation and gene expression by recognizing 2130 genome-wide differentiated methylated regions in 94 tissues of IPF patients. ${ }^{22}$ Cigarette smoking and aging have been identified as the main effectors of epigenetic modifications in IPF patients since both are responsible for IPF and DNA methylation. ${ }^{23,24}$ In recent studies, infections, especially by viruses (Epstein-Barr virus, Kaposi's sarcoma-associated herpesvirus, hepatitis B virus, human papillomavirus, HIV) and intracellular bacteria (Helicobacter pylori, chlamydia, mycobacteria, and salmonella) have also been found to be responsible for significant DNA methylation, histone methylation, histone acetylation, and other epigenetic changes. ${ }^{25}$ However, clinical studies have not been done to identify the relationship between virus-induced epigenetic changes and the development of IPF.

\section{INFECTIOUS CAUSES OF FIBROSIS}

The first association of viral infection and IPF was made in 1953 when many patients had a viral-type prodrome preceding respiratory symptoms. ${ }^{26}$ One of the first viruses that was suspected to be associated with IPF was hepatitis C virus (HCV), which causes fibrosis in the liver. However, in 1992, Japanese researchers Ueda et al. discovered a higher prevalence of HCV markers in IPF patients $(28.8 \%)$ than in control subjects. ${ }^{27}$ In 2008, Arase et al. established a strong association between $\mathrm{HCV}$ and idiopathic pulmonary fibrosis in a different way. They observed $6150 \mathrm{HCV}$ infected patients as test subjects and 2050 hepatitis $B$ virus (HBV) infected patients as controls for the development of IPF over a period of $8.0 \pm 5.9$ years in the HCV-group and $6.3 \pm 5.5$ years in the HBV group. In the HCV group, fifteen patients developed IPF compared to none in the HBV group. ${ }^{28}$ However, researchers from other countries have failed to replicate these results. Other investigators have also found an association of $\mathrm{HCV}$ with a range of fibrotic and non-fibrotic lung diseases. ${ }^{29}$

Other infectious causes of IPF have also been discussed in the literature. The most relevant evidence of IPF was found in patients with previous CMV, EBV, and less commonly Torque-Teno (Transfusion-Transmitted) (TT) virus. Both serological and pathological evidence of herpesvirus family, primarily CMV and EBV infection, was found in patients with IPF. Four studies have found anti-EBV, anti-herpes simplex virus (HSV-1), and anti-cytomegalovirus antibodies, primarily lgG. ${ }^{11}$ However, Manika et al. found evidence of $\operatorname{lgA}$ in $60 \%$ of patients with IPF, compared to $22 \%$ of control patients. ${ }^{31}$ Tang et al. noted the overwhelming influence of herpesviridae in the pathology of IPF, with about $97 \%$ of patients with IPF showing CMV, EBV, HHV7, or HHV8 infection(s), compared to $36 \%$ of the control patients. ${ }^{14}$ Cytomegalovirus, TTV, parainfluenza, rhinovirus, one case of HSV-1, and EBV have caused ARDS in patients with preexisting IPF, COPD, and asthma; acute exacerbations in IPF patients have been fatal. ${ }^{32-36}$

In 2002, when the SARS epidemic began, clinical and radiological evidence of residual pulmonary fibrosis was observed in many of the patients 
who survived. A one-month follow-up study on 258 patients suggested that $21.3 \%$ had pulmonary diffusion abnormality $\left(D_{L} C O\right)<80 \%$ of predicted. The study also showed that the patients who suffered from severe disease process and underwent aggressive treatment are the patients who showed signs of fibrotic changes. ${ }^{30} \mathrm{~A}$ one-year follow-up study done on 97 recovering SARS patients in Hong Kong showed reduced lung function and increased fibrotic changes in $27.8 \%$ of SARS survivors compared to the normal population. ${ }^{37,38}$ A two-year follow-up study also showed similar findings with a significant reduction in $D_{L} C O$, exercise capacity, and lung function tests. ${ }^{39}$ In a 15-year follow-up study on patients infected with SARS in 2003, $9 \%$ showed evidence of fibrosis following infection. However, that percentage decreased after one year, and it continued to decrease until the 15 -year follow-up in 2018 , remaining at $3.2 \%$. Ground glass opacities and intralobular and interlobular septum thickening were the most prominent fibrotic features seen in SARS patients at six months and 84 months, respectively. ${ }^{40}$ Similar to the SARS-CoV virus, when Middle East respiratory syndrome coronavirus (MERS-CoV) emerged in 2012, a retrospective study done on 36 MERS survivors showed significant radiographic changes in $36 \%$. Lung fibrosis involving one or multiple lobes was seen in 33\%, and groundglass opacities and pleural thickening were found in $5.5 \% .{ }^{41}$ Another pathologically similar virus, avian influenza A subtype $\mathrm{H} 5 \mathrm{~N}$, also caused diffuse alveolar damage (DAD) in patients, but was more acute and hemorrhagic in presentation. Reported cases showed an association between $\mathrm{H} 5 \mathrm{~N} 1$ infection and development of fibrosis. However, fibrosis associated with H5N1 was less organized than that with SARS. ${ }^{46}$

\section{Pathogenesis of fibrosis In SARS AND MERS}

During the SARS epidemic in 2003, researchers discovered from autopsies of fatal cases that the major pathological characteristic in SARS patients was $D A D$, which developed during the early phase of SARS development (7-10 days). Diffuse alveolar damage in SARS is characterized by an inflammatory infiltrate, extensive edema, exudative fluid accumulation, hyaline membrane formation, alveolar collapse, and desquamation. ${ }^{42}$ This phase is referred to as the acute phase. After ten days, in the medium phase of SARS development, DAD becomes organized through interstitial and alveolar fibrosis and type II pneumocyte hyperplasia. After 2-3 weeks, the late phase occurs, characterized by extensive fibrous organization and proliferation. ${ }^{43-50}$

The molecular mechanism responsible for fibrosis in SARS is consistent with the pathogenesis of IPF. TGF- $\beta$, one of the principal drivers of IPF, was elevated in the early phase of SARS-CoV infection. ${ }^{51}$ SARS-CoV-associated damage induced the release of numerous factors, such as extracellular matrix, acute phase reactants, and TGF- $\beta$. Thus, high level of TGF- $\beta$ was observed in damaged lung cells (including pneumocytes, bronchial epithelial cells, and monocytes/macrophages)..$^{52}$ However, whether it is caused by the virus itself is still unknown. ${ }^{50}$

Furthermore, the virus can induce high levels of serum and tissue TGF- $\beta$ expression and regulates the signal transduction of the TGF- $\beta$ pathway through the viral nucleocapsid $(\mathrm{N})$ protein. In alveolar epithelial cells and fibroblasts, overexpression of the $\mathrm{N}$ protein leads to TGF- $\beta$ pathway hyperactivation. This results in excess production of extracellular matrix (ECM) proteins, enhanced secretion of protease inhibitors, such as plasminogen activator inhibitor 1 (PAl-1) and tissue inhibitors of metalloproteinases (TIMP), reduced secretion of proteases, and myofibroblast differentiation and expansion. This ultimately results in increased deposition of the ECM protein and localization of myofibroblasts around active fibrosis sites. Thus SARS-CoV has been shown to cause lung fibrosis through its $\mathrm{N}$ protein and resultant activation TGF- $\beta$ pathway. ${ }^{53-55}$

Another mechanism involves angiotensin-converting enzyme-2 (ACE2) and angiotensin II (ANG-II) in the development of fibrosis. ACE2 proteins are expressed in various tissues in the human body, including the oral and nasal mucosa, nasopharynx, stomach, small intestine, colon, skin, lymph nodes, thymus, bone marrow, spleen, liver, kidney, and brain. However, it is most prominently expressed as a surface protein in the alveolar epithelial cells. ${ }^{56}$ ACE2 also has a protective role against fibrosis through negative regulation of 
local angiotensin level. SARS CoV has a spike protein on its cell surface that works as a receptor for ACE2. Interaction between the S1 subunit of the spike protein and the receptor promotes viral entry and inducing cell to cell fusion. ${ }^{56}$ Infection with the SARS CoV has been shown to reduce the ACE2 expression level in the lung epithelial cells. ${ }^{57}$ Reduced ACE2 can lead to an increase in the ANG-II levels, which are produced by fibroblasts and activated macrophages and stimulate the secretion of TGF- $\beta$ from the alveolar epithelial cells, possibly mediated by AGTR $1 .{ }^{56}$ TGF- $\beta$ itself can also stimulate ANG-II. Therefore, an "autocrine loop" may be present in the lung tissue.

In addition, ANG-II also activates downstream signaling mediators of the TGF- $\beta$ pathway, which are SMAD2 and SMAD4 proteins. ${ }^{57}$ It can also activate SMAD signaling in a TGF- $\beta$-independent but MAPKdependent manner. ${ }^{58}$ In addition, ANG-Il can also upregulate connective tissue growth factor (CTGF), promoting ECM deposition and lung fibrosis through the MEK/Erk pathway. ${ }^{59,60,62}$ Researchers have found that the ANG-II levels are significantly increased in the lung tissues after administration of the spike protein. ${ }^{61}$ Other mechanisms reported to have a role in SARS mediated lung fibrosis are upregulation of monocyte chemoattractant protein-1 (MCP-1) chemokine levels, ${ }^{63}$ signal transduction by mitogen-activated protein kinase (MAPK) through viral infection that results in cell differentiation and ECM production, phosphorylation and hyperactivation of $\mathrm{p} 38$, which results in actin organization, pulmonary myofibroblast activation, and $\alpha$-smooth muscle actin (SMA) expression. ${ }^{64-66}$ SARS- Co-V infection has been shown to cause EGFR upregulation and enhancement of lung disease in mouse models. ${ }^{67}$

MERS was found more frequently in type-II pneumocytes. Infection with MERS increased fibrotic growth factors, such as SMAD7 and FGF-2, in lung tissue. Both SMAD7 and FGF-2 are responsible for colocalizing caspase-3 expression that eventually results in epithelial cell apoptosis in MERS infected lung tissue ${ }^{68}$

\section{Clinical eVidence of Post-COVID fibrosis}

The first autopsy case report of post-COVID fibrosis was published online on July 28,2020 , in a breast cancer survivor woman in her 80s. Despite having no prior history of pulmonary infection, fibrotic lung with bilateral consolidations, septal thickening, traction bronchiectasis and infiltrative and parenchymal changes was found on the 39th day of her hospitalization with COVID-19 infection. ${ }^{69}$ Since then, many cases of postCOVID fibrosis have been identified. In a study done on 62 COVID-19 infected patients in Wuhan by Zhou et al., $21(33.9 \%)$ of the patients were found to have fibrotic changes, a finding more likely to be observed in the advanced phase of the disease (8-14 days after onset). ${ }^{70}$ Pan et al. also reported fibrotic changes in the CT scans of $17.5 \%$ of 63 patients during the acute phase of illness. ${ }^{71}$ Postmortem needle core biopsy findings in four patients who died of COVID-19 pneumonia also revealed features of fibroblastic proliferation and deposition of ECM in alveolar spaces along with diffuse alveolar damage. ${ }^{72}$ Patients affected by severe COVID-19 infection who later turned negative also had long-term lung dysfunction caused by pulmonary fibrosis. These patients ultimately benefited from lung transplantation. ${ }^{73}$

\section{Pathogenesis of fibrosis in COVID-19}

According to recent literature, COVID-19 causes diffuse lung damage through a severe inflammatory response. To mitigate this inflammatory response, regulatory pathways are activated that work toward healing the damaged tissue. Imbalance during this process leads to a fibrotic response of interstitial thickening, ground-glass opacities, irregular interface, coarse reticular pattern, and parenchymal band. ${ }^{74}$ Similar to the mechanism of infection in SARS-CoV, SARS-CoV-2 enters the host cells through membraneanchored ACE2. However, SARS-CoV-2 has a higher binding affinity to ACE2 receptors than that of SARSCoV. ${ }^{75}$ In addition, different proteases, such as transmembrane protease serine 2 (TMPRSS2) and other related proteases, e.g., ADAM17, cause ACE2 cleavage, facilitating the entry of SARS-CoV-2 in the alveolar epithelial cells. ${ }^{76}$ Compared to SARS-CoV, soluble ACE2 also has a protective effect against the binding of both SARS-CoV2 and pulmonary fibrosis induced by it. However, critically ill COVID-19 patients, especially those who were elderly, smokers, and diabetic, 
had upregulation of ACE2 in their lung tissue. This phenomenon leads to an increased number of viral entry points that ultimately lead to chronic internalization of ACE2 receptors, which increases ANG-II levels with a net increase in proinflammatory and profibrotic cytokines. ${ }^{77}$ Prolonged exposure to these cytokines leads to chronic pulmonary fibrosis, along with formation of stiff lung with less compliance. Stiff lung tissues have been found to induce altered cellular response and enhanced deposition of ECM proteins and glycosaminoglycans. All of these factors together lead to progressive pulmonary fibrosis in patients infected with COVID-19. ${ }^{78}$

\section{DISCUSSION OF TREATMENTS FOR COVID-19 INDUCED FIBROSIS}

Pulmonary fibrosis is a progressive and irreversible disease. However, with the right treatment, clinicians have a chance to slow the progression and improve the quality of life. If one can more effectively and efficiently treat the preceding lung disease (e.g., ARDS), this could slow or even stop the initiation of fibrosis in at-risk patients. After the fibrotic changes are underway, appropriate treatment might significantly slow the progression of the fibrosis with the goal of alleviating symptoms and improving morbidity and mortality in patients.

The ARDS Berlin criteria are used by clinicians to categorize and appropriately treat patients who develop ARDS regardless of the cause. However, ARDS secondary to COVID-19 may be different from ARDS secondary to other causes defined by the Berlin Criteria. ${ }^{79}$ For example, ARDS due to COVID-19 generally presents 8-12 days after the first symptoms appear; ${ }^{79}$ the ARDS Berlin Criteria state that to diagnose a patient with ARDS, the onset must be within one week of a known clinical change. In addition, the main damage caused by SARS-CoV-2 is to the alveolar epithelial cells rather than the capillary endothelial cells as it is in other causes of ARDS. ${ }^{79}$ Because ARDS appears to be a major factor in fibrosis development, these differences highlight the need to rethink the treatment given to COVID-19 patients with ARDS.

Although SARS-CoV-2 uses ACE2 receptors to enter cells and cause infection, ACE2 has been reported to have a protective role against lung fibrosis by downregulation of angiotensin 2 . Therefore, another potential treatment pathway targets angiotensin 2 to slow the progression of fibrosis. Waseda et al. studied mice with bleomycin-induced pulmonary fibrosis and found that the mice given an angiotensin 2 inhibitor had significantly lower lung fibrosis scores and TGFbeta levels. ${ }^{80}$ This study focused on drug-induced pulmonary fibrosis rather than infectious causes of pulmonary fibrosis; therefore, differences in treatment response require more study.

Pirfenidone and nintedanib are two antifibrotic drugs that have proven benefits in patients with IPF. They also demonstrate anti-inflammatory properties that can supposedly be used in the acute phase of COVID-19 pneumonia and ARDS. Pirfenidone can be used theoretically to attenuate LPS induced acute lung injury and resultant fibrosis through NLRP3 inflammasome suppression. ${ }^{81}$

Spironolactone has also shown significant results in the prevention of fibrosis. In animal models, it has antioxidant properties..$^{82}$ In several studies, a spironolactone showed alleviation of acute pneumonia through a reduced number of cells, such as lymphocytes, neutrophils, macrophages, and eosinophils, in the alveoli. ${ }^{82} \mathrm{Jin}$ et al. reported a significant role of spironolactone in the treatment of lung inflammation caused by bleomycin. ${ }^{83}$

In lung fibrosis, not only is there excess production of material secreted into the extracellular matrix, total ECM degradation is also thought to be reduced. ${ }^{84}$ Many factors are involved in ECM degradation, especially the plasminogen activator/plasmin system. Plasminogen is the precursor to plasmin, the major factor in fibrin degradation. The activity of plasminogen is regulated by plasminogen activator inhibitor 1 (PAI-1). In fibrotic lungs, PAl-1 expression is increased, hindering normal fibrin degradation. In animal studies, the deletion of the PAI-1 gene led to a reduced susceptibility to fibrosis. ${ }^{85}$ This could be a promising treatment in slowing the progression of ongoing fibrosis.

Other novel therapies, such as chitotriosidase 1 inhibitor with anti-inflammatory properties, tetrandrine (alkaloid that affects ROS production, calcium channels, and caspase pathways), mesenchymal stem cells (from human purified amniotic fluid that has anti-inflammatory and antifibrotic properties), 
hyperbaric oxygen therapy (that reduces the expression of $L-1 \beta, I L-6$, and TNF- $\alpha$, reducing ARDS induced fibrosis), lung transplantation, and rehabilitation is also being considered by researchers. ${ }^{86}$

\section{Conclusion}

The novel Coronavirus, SARS-CoV-2, and the disease it causes, COVID-19, are proving to be unlike any virus or disease studied thus far. The unprecedented infectivity and transmission has prompted worldwide studies and collaboration to find the best way to treat and prevent the disease. Unfortunately, because this disease is relatively new, there are not much data on long-term complications in patients. However, using the data and knowledge gained from similar viruses, we can predict potential complications and limit future problems. We are learning that there are some aspects of this disease that are not entirely consistent with what we previously thought (e.g., ARDS), and we should suspect that the usual treatment of similar diseases may not be as effective in treating this disease.

To date, most efforts to treat lung fibrosis target proinflammatory mediators, even though there is evidence to show that inflammation is not the primary cause of fibrosis. Future studies should explore the treatment potential of ECM degradation and the reduction of cytokine production and release. There will be many lasting consequences of this pandemic, but if we can mitigate the effects of even one potential consequence, we can improve patients' quality of life and reduce strain on the healthcare system.

Article citation: Ghose M, Patel M. A review of post infectious pulmonary fibrosis in the era of COVID-19 and potential treatment options. The Southwest Respiratory and Critical Care Chronicles 2021;9(40):37-46

From: Sir Salimullah Medical College (MG), Dhaka, Bangladesh; School of Medicine, Texas Tech University Health Sciences Center (MP), Lubbock, Texas

Submitted: 3/15/2021

Accepted: 6/27/2021

Reviewer: Rishi Raj MD

Conflicts of interest: none

This work is licensed under a Creative Commons

Attribution-ShareAlike 4.0 International License.

\section{REFERENCES}

1. WHO Director-General's opening remarks at the media briefing on COVID-19 - 11 March 2020. Accessed: October 1, 2020. Available from: https://www.who.int/dg/speeches/ detail/who-director-general-s-opening-remarks-at-themedia-briefing-on-covid-19-11-march-2020

2. Priniciples of Epidemiology in Public Health Service, Third Edition An Introduction to Applied Epidemiology and Biostatistics. Accessed: October 1, 2020. Available form: https:// www.cdc.gov/csels/dsepd/ss1978/lesson1/section11.html

3. COVID-19 Dashboard by the Center for Systems Science and Engineering (CSSE) at Johns Hopkins University. Accessed: October 1, 2020. Available from: https://coronavirus.jhu.edu/map.html

4. Coronaviruses. National Institute of Allergy and Infectious Disease. Accessed: October 1, 2020. Available from: https:// www.niaid.nih.gov/diseases-conditions/coronaviruses

5. Sgalla G, Iovene B, Calvello $\mathrm{M}$, et al. Idiopathic pulmonary fibrosis: pathogenesis and management. Respiratory research 2018;19(1):32.

6. Betensley A, Sharif R, Karamichos D. A systematic review of the role of dysfunctional wound healing in the pathogenesis and treatment of idiopathic pulmonary fibrosis. J Clin Med 2016;6(1):2.

7. Adams CJ, Kopp MC, Larburu N, et al. Structure and molecular mechanism of er stress signaling by the unfolded protein response signal activator IRE1. Front Mol Biosci 2019; 6:11.

8. Grimminger F, Günther A, Vancheri C. The role of tyrosine kinases in the pathogenesis of idiopathic pulmonary fibrosis. Eur Respir J 2015;45(5):1426-1433.

9. Barratt SL, Blythe T, Jarrett C, et al. Differential expression of VEGF-Axxx isoforms is critical for development of pulmonary fibrosis. Am J Respir Crit Care Med 2017;196(4):479-493.

10. Hamada N, Kuwano K, Yamada M, et al. Anti-vascular endothelial growth factor gene therapy attenuates lung injury and fibrosis in mice. J Immunol 2005;175(2):1224-1231.

11. Middle East Respiratory Syndrome. Centers for Disease Control and Prevention. Accessed: October 1, 2020. Available from: https://www.cdc.gov/coronavirus/mers/about/index.html

12. Kendall RT, Feghali-Bostwick CA. Fibroblasts in fibrosis: novel roles and mediators. Front Pharmacol 2014;5:123.

13. Strieter RM. What differentiates normal lung repair and fibrosis? Inflammation, resolution of repair, and fibrosis. Proc Am Thorac Soc 2008;5(3):305-310.

14. Spagnolo P, Cottin V. Genetics of idiopathic pulmonary fibrosis: from mechanistic pathways to personalised medicine. J Med Genet 2017;54(2):93-99.

15. Tsakiri K D, Cronkhite J T, Kuan P J, et al. Adult-onset pulmonary fibrosis caused by mutations in telomerase. 
Proceedings of the National Academy of Sciences of the United States of America 2007;104(18):7552-7557.

16. Nance T, Smith KS, Anaya V, et al. Transcriptome analysis reveals differential splicing events in IPF lung tissue [published correction appears in PLoS One. 2014;9(5):e97392]. PLoS One 2014;9(5):e97550.

17. DePianto DJ, Chandriani S, Abbas AR, et al. Heterogeneous gene expression signatures correspond to distinct lung pathologies and biomarkers of disease severity in idiopathic pulmonary fibrosis. Thorax. 2015;70(1):48-56.

18. Bridges RS, Kass D, Loh K, et al. Gene expression profiling of pulmonary fibrosis identifies Twist1 as an antiapoptotic molecular "rectifier" of growth factor signaling. Am J Pathol 2009;175(6):2351-2361.

19. Konishi K, Gibson KF, Lindell KO, et al. Gene expression profiles of acute exacerbations of idiopathic pulmonary fibrosis [published correction appears in Am J Respir Crit Care Med. 2009 Aug 15;180(4):380]. Am J Respir Crit Care Med 2009;180(2):167-175.

20. Boon K, Bailey NW, Yang J, et al. Molecular phenotypes distinguish patients with relatively stable from progressive idiopathic pulmonary fibrosis (IPF). PLoS One 2009;4(4): e5134.

21. Yang IV, Schwartz DA. Epigenetics of idiopathic pulmonary fibrosis. Transl Res 2015;165(1):48-60.

22. Yang IV, Pedersen BS, Rabinovich E, et al. Relationship of DNA methylation and gene expression in idiopathic pulmonary fibrosis. Am J Respir Crit Care Med 2014;190(11): 1263-1272.

23. Issa JP. Aging and epigenetic drift: a vicious cycle. J Clin Invest 2014;124(1):24-29.

24. Liu F, Killian JK, Yang M, et al. Epigenomic alterations and gene expression profiles in respiratory epithelia exposed to cigarette smoke condensate. Oncogene 2010;29(25): 3650-3664.

25. Paschos K, Allday MJ. Epigenetic reprogramming of host genes in viral and microbial pathogenesis. Trends Microbiol 2010;18(10):439-447.

26. Ueda T, Ohta K, Suzuki N, et al. Idiopathic pulmonary fibrosis and high prevalence of serum antibodies to hepatitis $\mathrm{C}$ virus. Am Rev Respir Dis 1992;146(1):266-268.

27. Arase Y, Suzuki F, Suzuki Y, et al. Hepatitis C virus enhances incidence of idiopathic pulmonary fibrosis. World J Gastroenterol 2008;14(38):5880-5886.

28. Irving WL, Day S, Johnston ID. Idiopathic pulmonary fibrosis and hepatitis C virus infection. Am Rev Respir Dis 1993 Dec;148(6 Pt 1):1683-4.

29. Meliconi R, Andreone P, Fasano L, et al. Incidence of hepatitis $\mathrm{C}$ virus infection in Italian patients with idiopathic pulmonary fibrosis. Thorax 1996;51:315-317.
30. Xie L, Liu Y, Xiao Y, et al. Follow-up study on pulmonary function and lung radiographic changes in rehabilitating severe acute respiratory syndrome patients after discharge. Chest 2005;127(6):2119-2124.

31. Moore BB, Moore TA. Viruses in idiopathic pulmonary fibrosis. etiology and exacerbation. Ann Am Thorac Soc. 2015; 12 Suppl 2(Suppl 2): S186-S192.

32. Tang YW, Johnson JE, Browning PJ, et al. Herpesvirus DNA is consistently detected in lungs of patients with idiopathic pulmonary fibrosis. J Clin Microbiol 2003;41(6):2633-2640.

33. Wootton SC, Kim DS, Kondoh Y, et al. Viral infection in acute exacerbation of idiopathic pulmonary fibrosis. Am J Respir Crit Care Med 2011;183(12):1698-1702.

34. Ushiki A, Yamazaki Y, Hama M, et al. Viral infections in patients with an acute exacerbation of idiopathic interstitial pneumonia. Respir Investig 2014;52(1):65-70.

35. Santos GC, Parra ER, Stegun FW, et al. Immunohistochemical detection of virus through its nuclear cytopathic effect in idiopathic interstitial pneumonia other than acute exacerbation. Braz J Med Biol Res 2013;46(11):985-992.

36. Kurai D, Saraya T, Ishii H, Takizawa H. Virus-induced exacerbations in asthma and COPD. Front Microbiol 2013; 4:293.

37. Hui DS, Joynt GM, Wong KT, et al. Impact of severe acute respiratory syndrome (SARS) on pulmonary function, functional capacity and quality of life in a cohort of survivors. Thorax 2005;60(5):401-409.

38. Hui DS, Wong KT, Ko FW, et al. The 1-year impact of severe acute respiratory syndrome on pulmonary function, exercise capacity, and quality of life in a cohort of survivors. Chest 2005;128(4):2247-2261.

39. Ngai JC, Ko FW, Ng SS, et al. The long-term impact of severe acute respiratory syndrome on pulmonary function, exercise capacity and health status. Respirology 2010;15(3):543-550.

40. Zhang P, Li J, Liu H, et al. Long-term bone and lung consequences associated with hospital-acquired severe acute respiratory syndrome: a 15-year follow-up from a prospective cohort study [published correction appears in Bone Res. 2020 Sep 21;8:34]. Bone Res 2020;8:8.

41. Das KM, Lee EY, Singh R, et al. Follow-up chest radiographic findings in patients with MERS-CoV after recovery. Indian J Radiol Imaging 2017;27(3):342-349.

42. Cheung OY, Chan JW, Ng CK, et al. The spectrum of pathological changes in severe acute respiratory syndrome (SARS). Histopathology 2004;45(2):119-124.

43. Gu J, Korteweg C. Pathology and pathogenesis of severe acute respiratory syndrome. Am J Pathol 2007;170(4):1136-1147.

44. Guo Y, Korteweg C, McNutt MA, Gu J. Pathogenetic mechanisms of severe acute respiratory syndrome. Virus Res 2008;133(1):4-12. 
45. Zuo W, Zhao X, Chen YG. SARS Coronavirus and Lung Fibrosis. Molecular Biology of the SARS-Coronavirus 2009;247-258. Published 2009 Jul 22.

46. Ng WF, To KF, Lam WW, et al. The comparative pathology of severe acute respiratory syndrome and avian influenza A subtype H5N1--a review. Hum Pathol 2006;37(4):381-390.

47. Nicholls J, Dong XP, Jiang G, et al. SARS: clinical virology and pathogenesis. Respirology 2003;8 Suppl (Suppl 1): S6-S8.

48. Tse GM, To KF, Chan PK, et al. Pulmonary pathological features in coronavirus associated severe acute respiratory syndrome (SARS). J Clin Pathol 2004;57(3):260-265.

49. Zuo W, Zhao X, Chen YG. SARS Coronavirus and Lung Fibrosis. Molecular Biology of the SARS-Coronavirus. 2009;247-258. Published 2009 Jul 22.

50. Pang BS, Wang Z, Zhang LM, et al. Dynamic changes in blood cytokine levels as clinical indicators in severe acute respiratory syndrome. Chin Med J (Engl) 2003;116(9):1283-1287.

51. Baas T, Taubenberger JK, Chong PY, et al. SARS-CoV virushost interactions and comparative etiologies of acute respiratory distress syndrome as determined by transcriptional and cytokine profiling of formalin-fixed paraffin-embedded tissues. J Interferon Cytokine Res 2006;26(5):309-317.

52. Liu RM, Vayalil $P$, Wang $S Q$, et al. TGF-betal induces concomitant lung fibrosis, decreased GSH and ascorbate concentrations, and increased PAI-1 gene expression. Free Radical Biol Med 2005;39:S37

53. Rube CE, Uthe $\mathrm{D}$, Schmid $\mathrm{KW}$, et al. Dose-dependent induction of transforming growth factor beta (TGF-beta) in the lung tissue of fibrosis-prone mice after thoracic irradiation. Int J Radiat Oncol Biol Phys 2000;47(4):1033-1042.

54. Zhao X, Nicholls JM, Chen YG. Severe acute respiratory syndrome-associated coronavirus nucleocapsid protein interacts with Smad3 and modulates transforming growth factor-beta signaling. J Biol Chem 2008;283(6):3272-3280.

55. Hamming I, Timens W, Bulthuis ML, et al. Tissue distribution of ACE2 protein, the functional receptor for SARS coronavirus. A first step in understanding SARS pathogenesis. J Pathol 2004;203(2):631-637.

56. Kuba K, Imai Y, Rao SA, Gao H, Guo F, Guan B, Huan Y, Yang P, Zhang YL, Deng W et al (2005) A crucial role of angiotensin converting enzyme 2 (ACE2) in SARS coronavirus-induced lung injury. Nat Med 11:875-879

57. Li W, Moore MJ, Vasilieva N, et al. Angiotensin-converting enzyme 2 is a functional receptor for the SARS coronavirus. Nature 2003;426(6965):450-454.

58. Molteni A, Wolfe LF, Ward WF, et al. Effect of an angiotensin II receptor blocker and two angiotensin converting enzyme inhibitors on transforming growth factor-beta (TGF-beta) and alpha-actomyosin (alpha SMA), important mediators of radiation-induced pneumopathy and lung fibrosis. Curr Pharm Des 2007;13(13):1307-1316.

59. Hao J, Wang B, Jones SC, et al. Interaction between angiotensin II and Smad proteins in fibroblasts in failing heart and in vitro. Am J Physiol Heart Circ Physiol 2000;279(6): H3020-H3030.

60. Su Z, Zimpelmann J, Burns KD. Angiotensin-(1-7) inhibits angiotensin II-stimulated phosphorylation of MAP kinases in proximal tubular cells. Kidney Int 2006;69(12):2212-2218.

61. Rodríguez-Vita J, Sánchez-López E, Esteban V, et al. Angiotensin II activates the Smad pathway in vascular smooth muscle cells by a transforming growth factor-betaindependent mechanism. Circulation 2005;111(19):2509-2517

62. Ponticos M, Holmes AM, Rajkumar V, et al. Connective tissue growth factor (CTGF) mediates extracellular matrix deposition in bleomycin-induced lung fibrosis via the MEK/ ERK signaling pathways. Arthritis Rheum 2003;48:S156

63. Emad A, Emad V. Elevated levels of MCP-1, MIP-alpha and MIP-1 beta in the bronchoalveolar lavage (BAL) fluid of patients with mustard gas-induced pulmonary fibrosis. Toxicology 2007;240(1-2):60-69.

64. Hu Y, Peng J, Feng D, et al. Role of extracellular signalregulated kinase, p38 kinase, and activator protein-1 in transforming growth factor-beta1-induced alpha smooth muscle actin expression in human fetal lung fibroblasts in vitro. Lung 2006;184(1):33-42.

65. Yoshida K, Kuwano K, Hagimoto N, et al. MAP kinase activation and apoptosis in lung tissues from patients with idiopathic pulmonary fibrosis. J Pathol 2002;198(3):388-396.

66. Mizutani T, Fukushi S, Ishii K, et al. Mechanisms of establishment of persistent SARS-CoV-infected cells. Biochem Biophys Res Commun 2006;347(1):261-265.

67. Venkataraman T, Coleman CM, Frieman MB. Overactive epidermal growth factor receptor signaling leads to increased fibrosis after severe acute respiratory syndrome coronavirus infection. J Virol 2017;91(12):e00182-17.

68. Yeung ML, Yao Y, Jia L, et al. MERS coronavirus induces apoptosis in kidney and lung by upregulating Smad7 and FGF2. Nat Microbiol 2016;1(3):16004.

69. Schwensen HF, Borreschmidt LK, Storgaard M, et al. Fatal pulmonary fibrosis: a post-COVID-19 autopsy case [published online ahead of print, 2020 Jul 28]. J Clin Pathol 2020; jclinpath-2020-206879.

70. Zhou S, Wang Y, Zhu T, Xia L. CT features of Coronavirus Disease 2019 (COVID-19) Pneumonia in 62 patients in Wuhan, China. AJR Am J Roentgenol 2020;214(6):1287-1294.

71. Pan $Y$, Guan H, Zhou S, et al. Initial CT findings and temporal changes in patients with the novel coronavirus pneumonia (2019-nCoV): a study of 63 patients in Wuhan, China. Eur Radiol. 2020;30(6):3306-3309. 
72. Tian S, Xiong Y, Liu H, et al. Pathological study of the 2019 novel coronavirus disease (COVID-19) through postmortem core biopsies. Mod Pathol 2020;33(6):1007-1014.

73. Chen JY, Qiao K, Liu F, et al. Lung transplantation as therapeutic option in acute respiratory distress syndrome for coronavirus disease 2019-related pulmonary fibrosis. Chin Med J (Engl). 2020;133(12):1390-1396.

74. Yu M, Liu Y, Xu D, et al. Prediction of the development of pulmonary fibrosis using serial thin-section ct and clinical features in patients discharged after treatment for COVID-19 Pneumonia. Korean J Radiol 2020;21(6):746-755.

75. Tseng YH, Yang RC, Lu TS. Two hits to the renin-angiotensin system may play a key role in severe COVID-19. Kaohsiung J Med Sci 2020;36(6):389-392.

76. Hoffmann M, Kleine-Weber H, Schroeder S, et al. SARSCoV-2 cell entry depends on ACE2 and TMPRSS2 and is blocked by a clinically proven protease inhibitor. Cell 2020; 181(2):271-280.e8.

77. Sparks MA, South A, Welling P, et al. Sound science before quick judgement regarding ras blockade in COVID-19. Clin J Am Soc Nephrol 2020;15(5):714-716.

78. Wigén J, Löfdahl A, Bjermer L, et al. Converging pathways in pulmonary fibrosis and Covid-19 - The fibrotic link to disease severity. Respir Med X 2020; 2:100023.

79. Li X, Ma X. Acute respiratory failure in COVID-19: is it “typical” ARDS?. Crit Care 2020;24(1):198.
80. Waseda Y, Yasui M, Nishizawa Y, et al. Angiotensin II type 2 receptor antagonist reduces bleomycin-induced pulmonary fibrosis in mice. Respir Res 2008;9(1):43.

81. Li Y, Li H, Liu S, et al. Pirfenidone ameliorates lipopolysaccharide-induced pulmonary inflammation and fibrosis by blocking NLRP3 inflammasome activation. Mol Immunol 2018; 99:134-144.

82. Lieber GB, Fernandez $X$, Mingo GG, et al. Mineralocorticoid receptor antagonists attenuate pulmonary inflammation and bleomycin-evoked fibrosis in rodent models. Eur J Pharmacol 2013;718(1-3):290-298.

83. Ji WJ, Ma YQ, Zhou X, et al. Spironolactone attenuates bleomycin-induced pulmonary injury partially via modulating mononuclear phagocyte phenotype switching in circulating and alveolar compartments. PLoS One 2013;8(11): e81090. Published 2013 Nov 19.

84. Liu RM. Oxidative stress, plasminogen activator inhibitor 1, and lung fibrosis. Antioxid Redox Signal 2008;10(2): 303-319.

85. Izuhara Y, Takahashi S, Nangaku M, et al. Inhibition of plasminogen activator inhibitor-1: its mechanism and effectiveness on coagulation and fibrosis. Arterioscler Thromb Vasc Biol 2008;28(4):672-677.

86. Lechowicz K, Drożdżal S, Machaj F, et al. COVID-19: The potential treatment of pulmonary fibrosis associated with SARS-CoV-2 infection. J Clin Med 2020;9(6):1917. 